\title{
Combining a Nanoprobing Setup with PFIB Sample Deprocessing
}

\author{
Andrew J. Smith ${ }^{*}$, Andreas Rummel ${ }^{1}$, Matthias Kemmler ${ }^{1}$ and Stephan Kleindiek ${ }^{1}$ \\ 1. Kleindiek Nanotechnik, Reutlingen, Germany. \\ * Corresponding author: andrew.smith@kleindiek.com
}

As the structure sizes in current-day semiconductor devices continue to shrink, the challenges in analyzing defects and failures in these products grow. Two crucial steps in the failure analysis workflow are deprocessing the sample to expose the devices exhibiting issues and employing electrical probes to locate and characterize the failures.

The established method for deprocessing samples entails the use of polishers and broad beam ion mills. These devices are not always able to reliably expose the desired layer of an Integrated Circuit device. Sometimes they lead to uneven surfaces or the small structures in recent technology nodes can be smeared. In addition, multiple iterations of polishing and moving to an SEM in order to inspect the progress with the help of Electron Beam Absorbed Current (EBAC) imaging are needed, resulting in a rather timeconsuming analysis workflow.

Thus, there is a trend to move the deprocessing step to a Plasma FIB (PFIB) [1]. These typically produce an ion beam from a Xe source and employ additional etching gases to assist in reliably and controllably removing material to a desired end-point. This method provides the means to site-specific and artefactfree access to the Region Of Interest (ROI).

Once the sample is deprocessed and ready for analysis, EBAC is used to non-destructively isolate Back End Of Line (BEOL) defects. EBAC works by touching a probe tip connected to a high-bandwidth amplifier down on a exposed metal line or metal plug on the sample [2]. As the electron beam is scanned over the sample surface, a current is registered on the tip every time the beam interacts with any metal structures connected to the line/plug in contact with the probe tip (Fig. 1). These currents are visualized as grey values during the scan and yield an image of current flow in the sample. By comparing the obtained imagery with the device's design schematics, the operator can determine where e.g. a short is located.

In order to further reduce the time required to complete device analysis, the nanoprobing system can be integrated into the PFIB microscope (Fig. 2), thus eliminating the need to switch between tools and allowing for the completion of the failure analysis procedure in a fraction of the time that it would take without this combination. Such a failure analysis workflow is depicted in Fig. 3.

Case studies show that the techniques described above are effective in revealing the root cause for specific failures in IC devices [3]. As shown in Fig. 4, the location of a bridging between two metal lines could be elucidated using PFIB to delayer the sample and EBAC to locate the issue. In this case, landing a probe tip on a certain contact resulted in a different, unrelated line appearing in the EBAC image. This finding lead the investigator to the short's location.

The implementation of this workflow also helps to minimize surface contamination and oxidation of the sample through exposure to ambient air, as the need to repeatedly remove the sample from the vacuum chamber for further processing is eliminated by combining all necessary tools in one instrument. Future 
work will expand the failure analysis toolkit to transistor probing on PFIB-delayered surfaces.

\section{References:}

[1] D Zudhistira et al., International Symposium of Testing \& Failure Analysis (2017).

[2] M Simon-Najasek et al., International Symposium for Testing and Failure Analysis, (2012).

[3] D Zudhistira, et al., International Symposium for Testing and Failure Analysis, (2018).

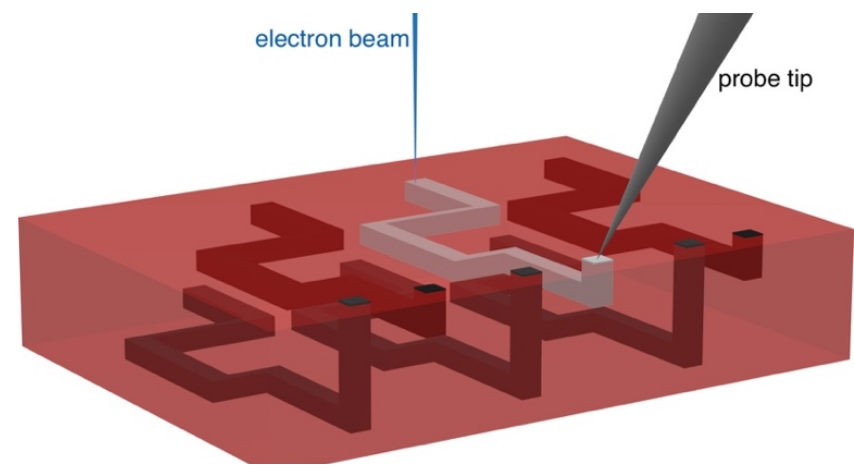

Figure 1: Schematic representation of EBAC.
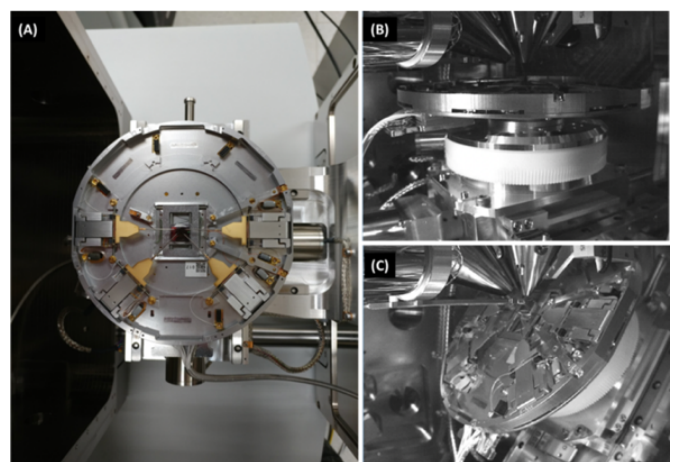

Figure 2: (a) Nanoprobing setup integrated into a PFIB microscope. (b) Chamberscope view of the configuration for EBAC. (c) Chamberscope view of the configuration for delayering.

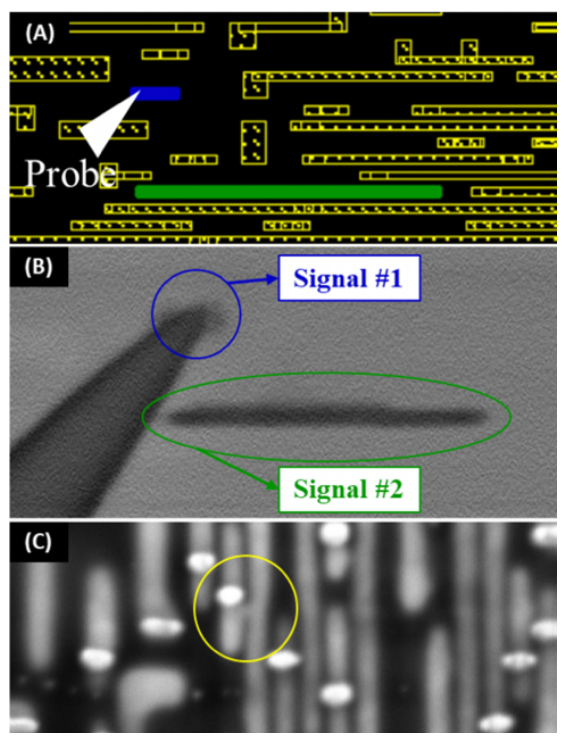

Figure 4: (a) CAD layout showing two different signals (green and blue). (b) EBAC at M4 on Signal\#1 causes Signal \#2 to light up, signifying short somewhere down the line. (c) Imaging at V3 reveals metal bridging between the two signals. 